\title{
DFT Based MMSE Equaliser in MIMO-OFDM
}

\author{
Richa Chouhan ${ }^{1}$, Rahul Dubey ${ }^{2}$, S G Kerhalker ${ }^{3}$ \\ ${ }^{\#}$ Oriental Institute of Science and Technology, Bhopal
}

\begin{abstract}
MIMO-OFDM is considered as a fast growing technology now days. OFDM is a multi carrier modulation technique in which the carriers are Orthogonal to each others as a result of which it provides high bandwidth efficiency and multiple carriers share the data among themselves. During the research equalizer is always a matter of strategies. When a signal is transmitted over a radio channel, it is subject to reflection, refraction and diffraction and also the type of modulation technique selected at transmitter. In this paper Bit Error Rate performance of OFDM under Rayleigh fading channel is analysed, and compared with Additive White Gaussian Noise Channel.
\end{abstract}

Keywords: OFDM, MIMO, ISI, MMSE, DFT

\section{INTRODUCTION}

Wireless Backhaul is the use of wireless communication system to get data from an end user to a node in a major network such as Internet or the proprietary network of a large business, academic institution and government agency [1]. In wireless backhaul, base nodes have the capability of relaying packets, and a few of them called core nodes serve as gateway which connects the wireless multihop network with the outside network. Various researches are running to improve the performance of wireless backhaul, one of the most commonly used approach is MIMO. A MIMO network is a wireless network made up of individual MIMO links. Multiple-input multiple-output (MIMO) wireless communication is one of the most promising technologies for improving the spectrum efficiency of wireless communication systems. It is well known that the use of MIMO antenna systems allows the channel capacity to scale in proportion to the minimum of the number of transmit and receive antennas in uncorrelated Rayleigh fading channels [2].

OFDM is similar to FDM but much more spectrally efficient by spacing the sub channel much more spectrally efficient by spacing much closer together. This is done by finding frequencies that are orthogonal, which means that are perpendicular in a mathematical sense, allowing the spectrum of each sub-channel to overlap another without Interfering with it. In the effect of this is seen as the required bandwidth is greatly reduced by removing guard bands and allowing signals to overlap .In order to demodulate the signal ,a discrete Fourier transform (DFT) is needed .Fast Fourier transform (FFT) chips are commercially available making this a relatively easy operation .

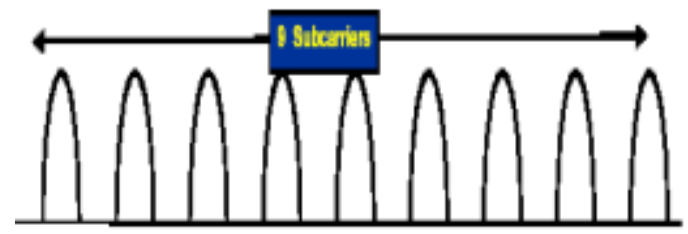

Fig 1. Frequency Division Multiplexing

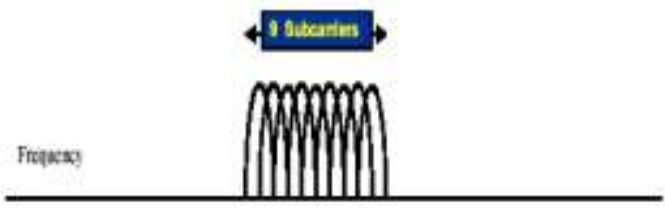

Fig 2. Orthogonal Frequency Division Multiplexing

A balanced linear equalizer is the Minimum Mean-Square Error (MMSE) equalizer, which does not usually eliminate ISI completely but instead minimizes the total power of the noise and ISI components in the output, therefore preferred in Orthogonal Frequency Division Multiplexing (OFDM). We want to solve the problem of Inter-symbol Interference (ISI) therefore the joint operation of OFDM with MMSE. In statistics and signal processing, MMSE estimator describes the approach, which minimizes the mean square error (MSE), which is a common measure of estimator quality. The term MMSE specifically 
refers to estimation in a Bayesian setting, since in the alternative frequency setting there does not exist a single estimator having minimal MSE [3].

\section{OFDM BASIC PRINCIPLES}

OFDM is a multicarrier transmission technique used in applications catering to both Wired and Wireless Communications To generate OFDM signals successfully the relationship between all carriers must be carefully controlled in order to maintain orthogonality. The spectrum required is first chosen based on the input data and the modulation scheme used (typically Differential BPSK, QPSK or QAM). Data to be transmitted is assigned to each carrier that is to be produced. Amplitudes and phases of the carriers are calculated based on the chosen scheme of modulation.

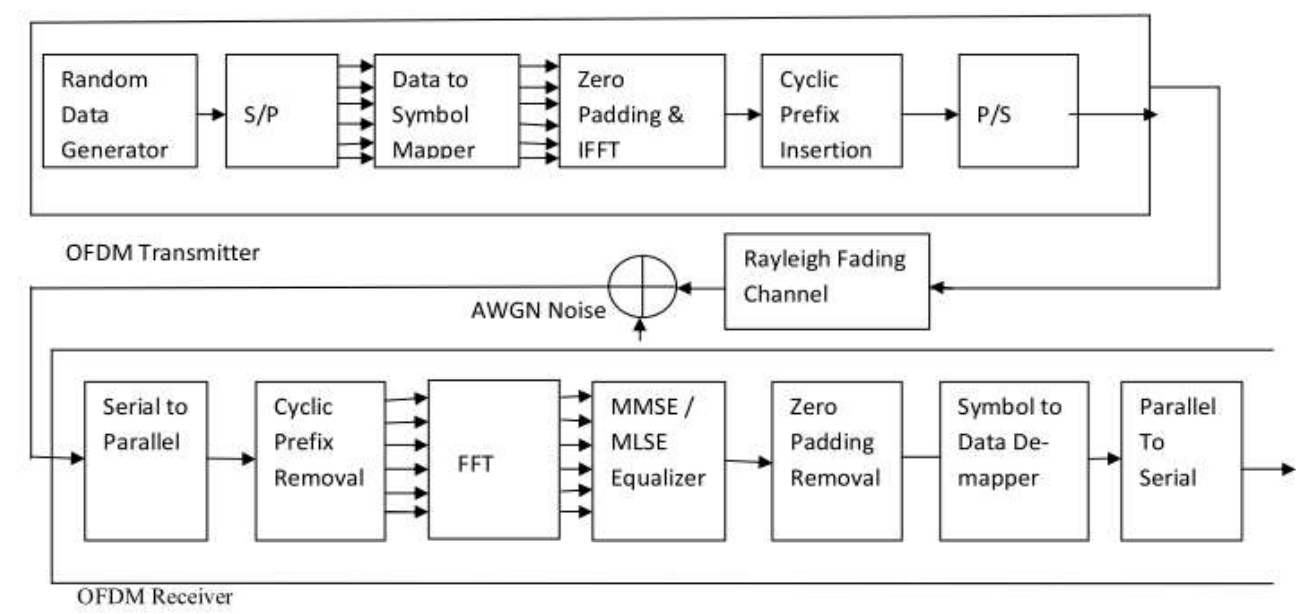

Fig 3. OFDM System Model

\subsection{Transmitter}

This subsection consists of following blocks [4]

\subsubsection{Random Data Generator:}

Random data generator is used to generate a serial random binary data .This binary Data stream models the raw information that going to be transmitted. The serial binary data is then fed into OFDM transmitter.

\subsubsection{S/P converter:}

The input serial binary data stream is grouped into word size required for transmission in this each word, and word is converted into parallel stream. Each stream is used to modulate one carrier out of group of orthogonal carrier.

\subsubsection{Data to symbol Mapper:}

This block does modulation like BPSK, QPSK, QAM, \& 16QAM .The data on each symbol is mapped to a particular phase based on the modulation method used .Each one the phase is assigned a unique pattern of binary bit .Usually each phase encodes an equal number of bits.

\subsubsection{Zero-padding and IFFT:}

The IFFT converts frequency domain data into the time domain signal. Prior to IFFT mapping zeropadding is performed to adjust the IFFT bit size of length. Zero padding is used because the number of subcarriers may be less then bit size. Let $\mathrm{Xp}(\mathrm{k})$ is the input Vector to IFFT block and $\mathrm{k}$ varies from 0 to $\mathrm{N}$ 1 Where $\mathrm{N}=64$. Out put of IFFT is given by

$$
\mathbf{X}_{\mathbf{P}}(\mathbf{n})=\operatorname{IFFT}\left[\mathbf{X}_{\mathbf{P}}(K)\right]
$$

\subsubsection{Cyclic Prefix:}

It is a cyclic extension of an OFDM symbol to eliminate ISI effect on original OFDM symbol. The length of cyclic prefix is chosen $1 / 4$ of the length of symbol. The cyclic prefix adds time over head decreasing the overall spectral efficiency of the system.After the cyclic prefix has been added [5] 


\subsection{Channel model:}

\subsubsection{AWGN Channel:}

Additive white Gaussian Noise (AWGN) is a channel model in which the only impairment to communication is a linear addition of wideband or white noise with a constant spectral density (expressed as watts par hertz of bandwidth) and a Gaussian distribution of amplitude. The model does not account for fading, frequency, selectivity, interference, nonlinearity or dispersion. However, it produces simple and tractable mathematical models which are useful for gaining insight into the underlying behavior of a system before these other Wideband Gaussian noise comes from many natural sources, such as the thermal vibrations of atoms in conductors (referred to as thermal noise or JohnsonNyquist noise), shot noise, black body radiation from the earth and other warm objects and from celestial sources such as the sun [6]. AWGN does not work will thus the more specified model are used. Fading is deviation of the attenuation that a carried modulated telecommunication signal experiences over certain propagation media. A fading channel is communication Rayleigh fading is caused by multipath reception really fading is statistical model for the effect of propagation environment on a radio signal such as is used by wireless devices.

\subsubsection{Rayleigh Fading Environment:}

Rayleigh and Rician fading channels are useful models of real-world phenomena in wireless communications. These phenomena include multipath scattering effects, time dispersion, and Doppler shifts that arise from relative motion between the transmitter and receiver. The figure below depicts direct and major reflected paths between a stationary radio transmitter and a moving receiver. The shaded shapes represent reflectors such as buildings.

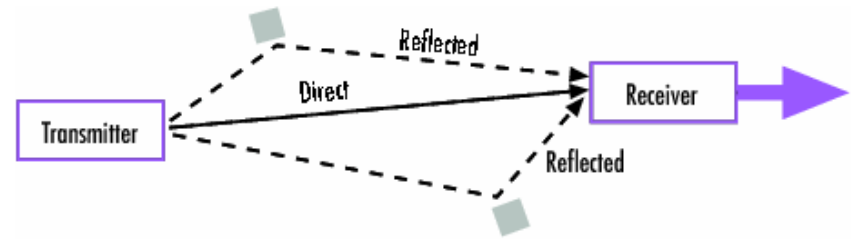

The major paths result in the arrival of delayed versions of the signal at the receiver. In addition, the radio signal undergoes scattering on a local scale for each major path. Such local scattering is typically characterized by a large number of reflections by objects near the mobile. These irresolvable components combine at the receiver and give rise to the phenomenon known as multipath fading. Due to this phenomenon, each major path behaves as a discrete fading path. Typically, the fading process is characterized by a Rayleigh distribution for a nonline-of-sight path and a Rician distribution for a line-of-sight path.

The relative motion between the transmitter and receiver causes Doppler shifts. Local scattering typically comes from many angles around the mobile. This scenario causes a range of Doppler shifts, known as the Doppler spectrum. The maximum Doppler shift corresponds to the local scattering components whose direction exactly opposes the mobile's trajectory.

\subsection{Receiver:}

The receiver does the reverse in contrast to the transmitter. Firstly the serial output channel is a converted into parallel stream and then cyclic prefix bits are removed from it.Then FFT of Each symbol is performed .To remove these channel effect MMSE and MLSE is performed equalized output is converted back to data words by demodulator the phenomena are considered data words are then multiplexed to get the original data.

\section{EQUALIZER:}

Equalizer [7] is a digital filter that provides an approximate inverse of channel frequency response. Equalization is to mitigate the effects of ISI to decrease the probability of error that occurs without suppression of ISI, but this reduction of ISI effects has to be balanced with prevention of noise power enhancement.

\subsection{Adaptive equalization:}

Adaptive equalizer is an equalizer that automatically adapts to time-varying properties of the communication channel. It is frequently used with coherent modulations such as phase shift keying, mitigating the effects of multipath propagation and Doppler spreading. 


\subsection{Blind equalization:}

Equalizer minimizes the error between actual output and desired output by continuous Blind is a digital signal processing technique in which the transmitted signal is inferred from the received signal. While making use only of the transmitted signal statistics.

\subsection{Minimum Mean Square Error Equalizer (MMSE):}

In statistics and signal processing, a minimum mean square error (MMSE) estimator describes the approach which minimizes the mean square error (MSE), which is a common measure of estimator quality.

The term MMSE more specifically refers to estimation in a Bayesian setting with quadratic cost function. The idea is that we often have some prior information about the parameters to be estimated, instead of knowing absolutely nothing about it. This prior information is captured by the prior probability density function of the parameters and allows us to make better posterior estimates as more observations become available. Thus unlike non-Bayesian approach where parameters of interest are assumed to be deterministic, but unknown constants, the Bayesian estimator seeks to estimate a parameter that is itself a random variable [8].

Let $\mathrm{X}$ be an unknown random variable, and let $\mathrm{Y}$ be a known random variable (the measurement). An estimator $\mathrm{X}_{\mathrm{e}}$ is any function of the measurement $\mathrm{Y}$, and its MSE is given by

$$
\mathrm{MSE}=\mathbf{E}\left\{\left(\mathbf{X}_{\mathrm{e}}-\mathbf{X}\right)^{2}\right\}
$$

where the expectation is taken over both $\mathrm{X}$ and Y.The MMSE estimator is then defined as the estimator achieving minimal MSE.

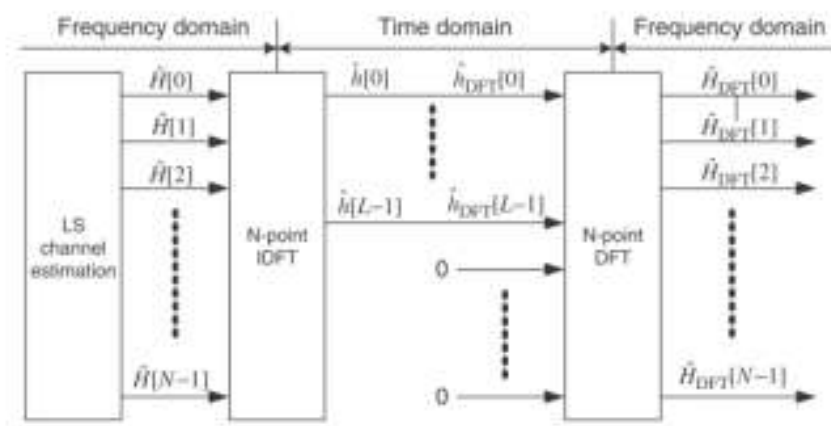

Fig 4 DFT based Estimation

The DFT-based channel estimation technique has been derived to improve the performance of LS or MMSE channel estimation by eliminating the effect of noise outside the maximum channel delay.

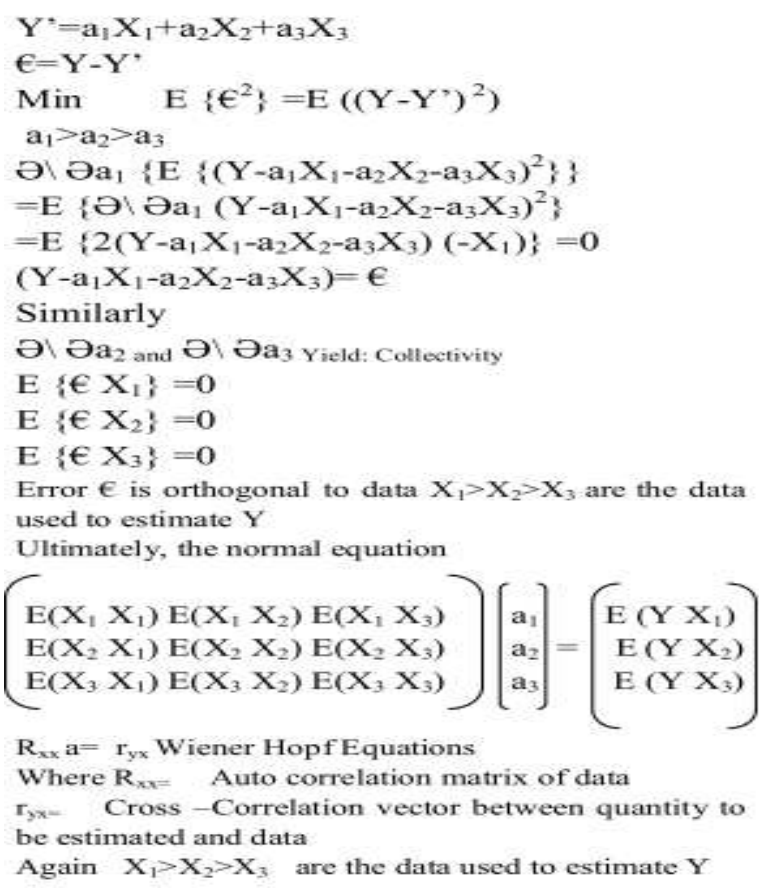




\section{SIMULATION AND RESULT}

Simulation parameters: Simulation parameters chosen for the model of OFDM transceiver re listed in Table 1.

\begin{tabular}{|c|l|l|}
\hline S.No & Parameter Used & \multicolumn{1}{c|}{ Value } \\
\hline 1 & $\begin{array}{l}\text { Carrier } \\
\text { Modulation }\end{array}$ & QPSK, BPSK, QAM \\
\hline 2 & Data Sub Carrier & $\mathbf{6 4 , 5 2}$ \\
\hline 3 & IFFT & 64 \\
\hline 4 & CP Length & $\mathbf{1 6}$ \\
\hline 5 & Channel & AWGN, Rayleigh \\
\hline 6 & Bandwidth & 20 MHz \\
\hline 7 & Equaliser & MMSE, MMSE with DFT \\
\hline
\end{tabular}

Table 1. Simulation Parameter for MIMO OFDM

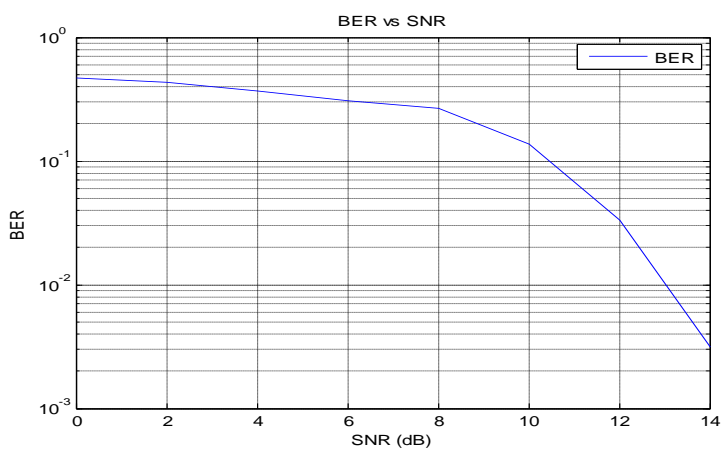

Fig 5. BER performance of OFDM under AWGN channel

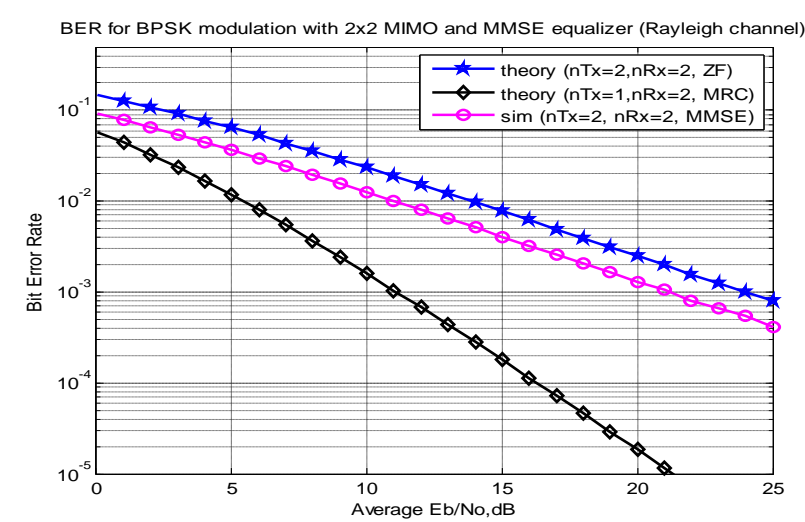

Fig 6. BER performance of MIMO OFDM under Rayleigh Fading Channel

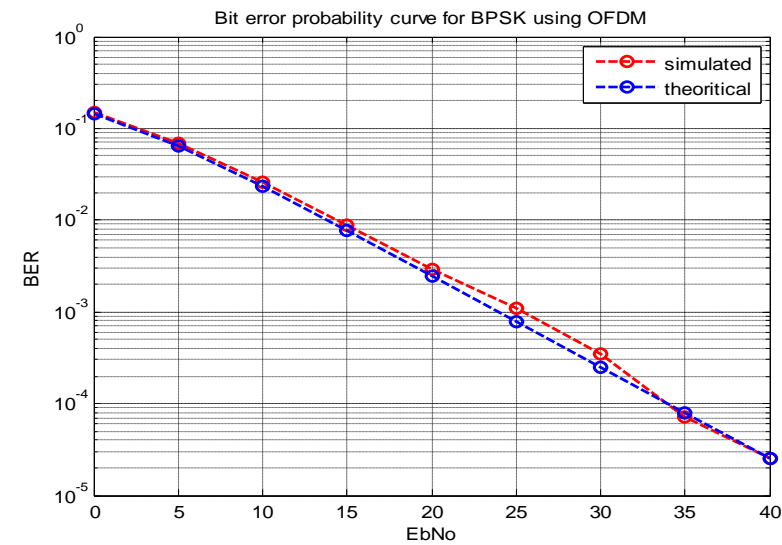

Fig 7 BER Performance of MIMO OFDM for BPSK modulation scheme 

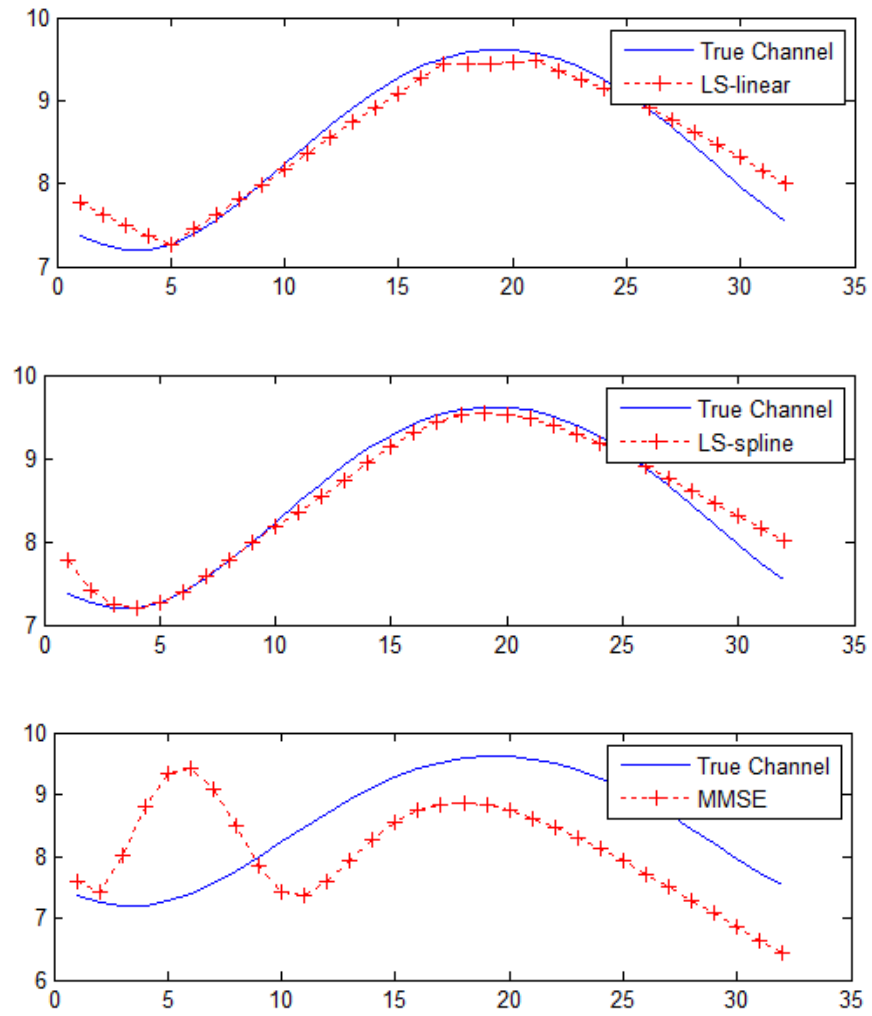

Fig 8. Channel Estimation with MMSE only
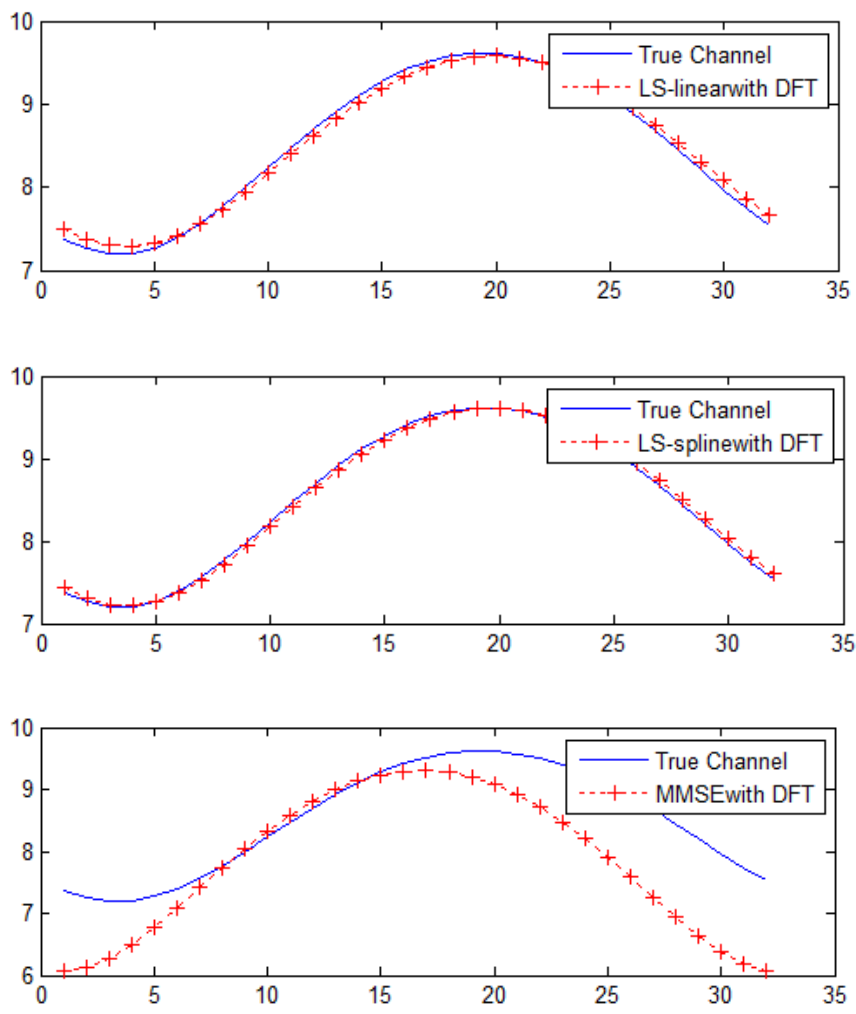

Fig 9 Channel estimation with MMSE-DFT

\section{CONCLUSION}

The performance of MIMO-OFDM under various channels is analysed using MMSE Equilizer with DFT. The paper compares the performance of un equalized systems with the equalized system. The bit error performance is improved. 2 Transmitting antenna, and 2 receiving antenna is used in the experimental 
simulation to judge the performance of MIMO system. Zero Forcing equaliser performs well only in theoretical assumptions that are when noise is zero. Its performance degrades in mobile fading environment. Minimum Mean Square Error (MMSE) equalizer uses LMS (Least Mean Square) as criterion to compensate ISI. The MMSE equalizer results in around $3 \mathrm{~dB}$ of improvement when compared to zero forcing equalizer.

\section{REFERENCES}

[1] E. M. Mohamed, D. Kinoshita, K. Mitsunaga, Y. Higa, and H.Furukawa, -MIMO based Wireless Backhaul $\square$, ICUMT-2010, pp.202-209, IEEE.

[2] G. J. Foschini and M. J. Gans, -On limits of wireless communications in a fading environment when using multiple antennas, Wireless Personal Commun., vol. 6, no. 3, pp. 311-335, Mar. 1998.

[3] Sikarwar. N, "IMPROVEMENT IN BER PERFORMANCE BY MMSE EQUALIZER WITH MIMO OFDM", International Journal for Research in Science \& Advanced Technologies, Vol .1, ISSN: 2319-2690.

[4] Boumard Sand Mammela A "Channel estimation versus equation in an OFDM WLAN system" in proc. Vehicular Technology Conference, pp 653-657, 2001.

[5] B. Muquet, Zwang, G.B. Giannakis, M.de Courville and P.Duhamel ,"Cyclic prefixing or zero padding for wireless multicarrier transmission,"IEEE Trans. On Comm., vol.50.no 12, pp.2136-2148, Dec 2002.

[6] P.Banelli, 'OFDM signal in fading channels" IEEE Trans, on wireless comm. vol.2, no. 2, pp.284-293, mar.2003.

[7] M.X.Chang and Yu T.Su ," performance analysis of Equalized OFDM systems in Rayleigh fading” IEEE Trans. On wireless comm. Vol. 1 no.4. pp.721-732.oct2002.

[8] Kuchi, K, "MMSE-Prewhitened-MLD Equalizer for MIMO DFT- precoded-OFDMA", Wireless Communications Letters, IEEE, pp.328-331, May-2012. 